\title{
Associations et comités de village mexicains de Californie
}

\section{Emmanuelle Le Texier}

\section{(2) OpenEdition \\ 12 Journals}

Édition électronique

URL : https://journals.openedition.org/remi/2033

DOI : $10.4000 /$ remi.2033

ISSN : $1777-5418$

Éditeur

Université de Poitiers

\section{Édition imprimée}

Date de publication : 1 décembre 2004

Pagination : 163-177

ISBN : 2-911627-38-5

ISSN : 0765-0752

Référence électronique

Emmanuelle Le Texier, "Associations et comités de village mexicains de Californie », Revue européenne des migrations internationales [En ligne], vol. 20 - n³ | 2004, mis en ligne le 25 septembre 2008, consulté le 16 avril 2022. URL : http://journals.openedition.org/remi/2033 ; DOI : https://doi.org/ 10.4000/remi.2033

Ce document a été généré automatiquement le 16 avril 2022.

(c) Université de Poitiers 


\title{
Associations et comités de village mexicains de Californie
}

\author{
Emmanuelle Le Texier
}

1 Les négociations entre le Mexique et les États-Unis, entreprises en 2000, pour statuer sur la régularisation des sans papiers, sur un programme de travailleurs saisonniers et plus généralement sur des politiques migratoires, restent au point mort. Cet échec, en partie dû à l'obsession sécuritaire américaine dans le contexte des attentats du 11 septembre 2001, a provoqué la démission du ministre mexicain des Affaires Étrangères Jorge Castañeda. C'est à l'initiative du président français lors du sommet du G-8 tenu à Évian que Vicente Fox a pu rencontrer son homologue George W. Bush et relancer le dialogue sur l'enjeu brûlant de l'immigration. La reprise des discussions bilatérales en juin 2003 et le projet d'une législation migratoire américaine pour fin 2004 (Land Border Security and Immigration Improvement Act) soulignent l'importance de l'agenda mexicain pour les États-Unis, tant en matière de politique étrangère que domestique (Le Texier, $2004: 88-96)$.

2 Le recensement de 2000 montre que les Latinos constituent la première minorité du pays : $12,3 \%$ de la population totale (35,4 millions). Les Latinos d'origine mexicaine, toutes générations confondues, composent à eux seuls plus de 20,6 millions d'individus (U.S Census Bureau, 2001). Depuis le début des années 1990, l'immigration mexicaine aux États-Unis, légale et illégale, forme le plus important des flux migratoires. Les flux s'agrègent aux populations établies aux États-Unis depuis le Traité de Guadalupe Hidalgo qui marque l'annexion d'une partie du Mexique au milieu du XIXe siècle, puis à celles venues par différentes vagues tout au long du siècle dernier. L'immigration mexicaine s'est accélérée à la fin des années 1970 (Odgers, 2003 : 59-78). Depuis 1994, et en dépit de la militarisation de la frontière dans le but de freiner l'immigration illégale, chaque année de 400000 à 500000 migrants parviennent à traverser « vers le Nord " clandestinement ${ }^{1}$.

3 La superposition de ces flux migratoires, légaux et clandestins, a favorisé la création de réseaux, en particulier entre les États d'origine les plus "exportateurs" (Oaxaca, Guerrero, Michoacán, Zacatecas) et les États de destination les plus «importateurs » 
(Californie, Illinois). Au point que certains auteurs parlent de l'édification symbolique de nouvelles régions comme Oaxacalifornia - Oaxaca et Californie - ou de nouvelles villes telle Chula Juana - Chula Vista et Tijuana - (Kearney, 1995). Selon la théorie cumulative $^{2}$, l'existence de ces flux entre communautés d'origine et localités de destination provient de l'accumulation du capital social qui élargit la base des candidats à l'immigration à mesure que les coûts de départ diminuent. L'émergence de "villes-tandems" favorise alors non seulement la concentration géographique des migrants dans le pays d'accueil mais aussi la formation de réseaux de solidarité. Ceux-ci épousent deux formes principales.

4 Au plan individuel, la solidarité des immigrés mexicains envers leurs familles s'exprime dans l'envoi de biens matériels mais surtout de remises (remesas) : l'équivalent de 11 milliards de dollars en 2003, deuxième source de devises du Mexique après le pétrole. $\mathrm{Au}$ plan collectif, cette solidarité se concrétise dans la création de comités de villages (hometown associations), plus de 500 au total concentrés en Californie, Texas et Illinois. Ces associations à but non lucratif ont pour objectif de soutenir le développement économique et social de la ville ou du village d'origine. Les activités développées par les comités concernent principalement le secteur sanitaire et éducatif $(60 \%)$ et la construction d'infrastructures (32\%) qui prennent la forme de rénovation d'églises, construction de centres de loisirs ou encore embellissement des villes. En 2002, l'investissement financier des comités de villages équivaut à moins de $5000 \$$ dans $40 \%$ des projets et de $5000 \$$ à $10000 \$$ dans $30 \%$ des cas. Au total, ces clubs ont contribué à hauteur de 30 millions de dollars à des programmes de développement en 2003. Enfin, parmi les États les plus receveurs de cette aide se trouvent Zacatecas (un tiers du total), puis les États de Jalisco, Guanajuato et Michoacan (Orozco et Lapointe, 2003). Les comités se sont donc constitués en force économique inégalée, participant du développement global des villages d'origine. Mais au-delà de leur vocation philanthropique, ils ont également acquis un rôle politique croissant.

Plus que de simples organisations binationales de migrants, les comités de villages développent, quand ils sont organisés en fédérations régionales, des stratégies politiques transnationales. L'examen détaillé de la naissance de cette forme de transnationalisme "par le bas" (Smith et Guarnizo, 1998) montre toutefois le rôle essentiel de l'État mexicain dans l'institutionnalisation "par le haut» de ces organisations. En effet, depuis la fin des années 1980, les associations de villages ont commencé à se regrouper au sein de fédérations régionales dont les fonctions vont bien au-delà de l'aide matérielle et économique. En Californie, les douze fédérations régionales, en majorité basées à Los Angeles (Federación de Zacatecanos, Clubs de Michoacanos, Frente Indígena Oaxaqueño Binacional, Clubs de Sinaloenses, Nayaritas en California, Federación de Guerrero, Clubs de Jalicienses, etc.), sont devenues les interlocuteurs privilégiés des États, du gouvernement et des hommes politiques mexicains. Il s'agira alors de différencier deux types de fédérations en fonction de leur rapport de coopération ou de conflit avec l'État d'origine ${ }^{3}$.

\section{Des comités de village aux fédérations régionales}

6 La création des comités de village en Californie n'est ni une exception, ni une nouveauté. Les migrants qui s'installent dans un nouvel environnement tentent de faire jouer la solidarité dans le pays d'accueil. Les premières associations d'immigrés 
mexicains aux États-Unis se sont d'ailleurs créées dès le XIX ${ }^{e}$ siècle pour défendre les droits des travailleurs agricoles. Les mutualistas avaient aussi pour but de participer à la préservation de l'héritage culturel mexicain et d'aider les familles restées au Mexique.

Les associations, clubs ou comités de village au nombre de 200 à 220 en Californie se sont formés afin de soutenir les communautés d'origine par des projets de développement local. À Los Angeles, ces clubs seraient au nombre de 170 pour 12 fédérations ${ }^{4}$. Les réseaux établis ont tendance à générer de la solidarité dans le pays d'accueil entre immigrés confrontés à la même situation, et envers les proches restés au pays. "Los del Norte » (ceux du Nord), «Los de afuera » (ceux de l'extérieur), «Los Mexicanos ausentes» (les Mexicains absents) contribuent à l'investissement et au développement local. Ces formes organisées de solidarité vont de l'envoi de matériel scolaire ou de maillots pour l'équipe locale de foot-ball, au financement d'infrastructures ou de rénovation de monuments religieux et administratifs. Par exemple, les municipios de Villanueva et Jerez (État de Zacatecas) ont reçu respectivement un million et six cent mille dollars afin de construire des routes, écoles et un système d'irrigation dans les villages de San Juan del Centro, Colonia Felipe Angeles et Boca de Rivera (Orozco et Lapointe, 2003). De même, dans le village de Santa Rosa à Oaxaca, les bâtiments de l'école publique, les routes pavées, l'électricité, les puits et l'arrivée d'ordinateurs sont le fruit de la coopération entre le Club de Santa Rosa basé à Oceanside (nord du comté de San Diego) et l'équipe municipale. Les immigrés, principalement travailleurs agricoles, soulignent que cet effort est une question vitale. Juan, membre de ce club et établi depuis 13 ans aux États-Unis, signale ainsi la nécessité de participer au développement local pour pallier les lacunes d'un Etat qui ne remplit pas ses fonctions :

«Por eso trabajo para el desarrollo de Santa Rosa: es un pueblo de unas 500 personas porque de alla se fueron casi 1400 personas que se vinieron de este lado. Desde que creamos el Club, mandamos dinero y programas y ya instalamos el agua potable, el alumbrado público, el $95 \%$ de las carreteras las hicimos nosotros, pavimentamos todas las calles. Porque el gobierno no cumple con sus promesas ni con sus deberes, entonces hay cooperación entre el pueblo de Santa Rosa y nosotros $»^{5}$.

8 Les comités de village les plus actifs fonctionnent sur le modèle de communautés d'échange des deux côtés de la frontière (flux monétaires, de biens, d'informations et d'individus) ${ }^{6}$. Le champ des activités s'étend de la solidarité économique aux domaines sociaux et culturels. Les traditionnels concours de Reine de beauté peuvent ainsi faire gagner des candidates qui résident aux États-Unis et se rendent au village d'origine uniquement pour la compétition. Les célébrations religieuses ou folkloriques constituent souvent des moments clefs du retour des immigrés qui en profitent pour superviser et évaluer les projets en cours.

9 Si les associations d'immigrés ont été analysées en fonction des mécanismes d'intégration et d'assimilation, le phénomène de solidarité avec la communauté d'origine pourtant ancien (Guarnizo, 2001) n'a été que récemment étudié. De plus, la transnationalisation des activités de solidarité des migrants a des significations et des implications différentes aujourd'hui. En effet, les contextes locaux et globaux ont changé (hostilité du pays d'accueil, pression des migrants, intérêt stratégique du pays d'origine). Les réseaux ont acquis une autre ampleur du fait de la globalisation, de la diminution des coûts de communication et de transports, des avancées technologiques et médiatiques. Ce sont donc les approches de l'immigration en termes de processus, de 
circuits migratoires (Rouse, 1994), de réseaux cumulatifs (Massey et Alarcón, 1994) ou de communautés migrantes transnationales (Schiller, Basch et Blanc-Szanton, 1995) qui ont renouvelé les analyses de ce phénomène sous l'angle transnational. Le tournant provient de la théorisation de Schiller, Basch et Blanc-Szanton (1995: 27) qui définissent ainsi les communautés migrantes transnationales: "(they) are grouping of immigrants who participate on a routine basis in a field of relationships, practices, and norms that include both places of origin and places of destination ». La littérature a longtemps privilégié l'étude univoque du rôle de l'État d'accueil dans l'adaptation et dans l'intégration politique des immigrés. Dans ce nouveau paradigme, le rôle de l'État d'origine dans la mobilisation des migrants recouvre une dimension essentielle. Il s'agit donc de comprendre en quoi les fédérations mexicaines servent le projet nationaliste de l'État mexicain tout en cherchant à préserver leur autonomie.

\section{L'institutionnalisation des mécanismes de coopération}

10 Il faut tout d'abord distinguer entre le niveau informel et le niveau institutionnalisé des activités migrantes transnationales, à partir de la transition qui s'est opérée dans les années 1980 et 1990. L'institutionnalisation de la solidarité migrante a répondu d'une part, à la nécessité d'assurer le maintien des structures de pouvoir au plan local au Mexique et, d'autre part, à l'intérêt croissant de l'État mexicain envers sa diaspora : « In response to transnationalization of political life by immigrants, (the State is) attempting both to control and channel this activity and to mute its potential to disrupt domestic politics » (Guarnizo et Smith, $1998: 223$ ).

11 La canalisation de cette forme de transnationalisme par le bas se réalise à deux échelles. Au plan des États qui composent la République mexicaine, le poids économique de certains comités est en effet venu perturber les équilibres politiques et sociaux d'origine. L'influence qu'exercent les associations de village sur l'allocation des ressources a fait prendre conscience de l'impérative formalisation des échanges des deux côtés de la frontière. Les élus locaux et étatiques ont ainsi impulsé l'institutionnalisation des aides et des statuts par le biais de la création ou de la mise en place concertée de fédérations régionales. Le cas le plus parlant est celui de la Federación de Zacatecanos fondée en 1985 à Los Angeles. Avec plus d'un million de migrants aux États-Unis, l'État de Zacatecas est l'un des plus exportateurs d'immigration. La fédération de cet État regroupe 43 associations de village (Goldring, 1999: 34-51). Conscient du poids des migrants dans le développement de l'État de Zacatecas, le gouverneur Borrego a entamé l'institutionnalisation des clubs et de la Fédération. En 1985, après une visite à Los Angeles, il a institué le Jour des Zacatecanos Absents et le Programme pour les Zacatecanos à l'étranger. Son successeur, le gouverneur Romo, a renforcé cette institutionnalisation par le Programme 2X1. Pour chaque dollar apporté par les clubs dans un projet de développement local, l'État contribuait à même hauteur. En 1994, l'apport total était de 2 millions de dollars, pour près de 200 projets menés. Dépassé par les financements de l'extérieur, l'État a alors dû créer un seuil maximal à ses apports et remplacer le Programme 2X1 par Ramo 26. Comme le note Victor Manuel Sánchez, président de la Fédération de Zacatecanos, l'institutionnalisation a permis de rendre plus efficace l'aide apportée aux différentes villes, de définir une hiérarchie des priorités de développement à l'échelle de l'État et 
d'accroître l'investissement global. La Fédération s'est en outre élevée au rang de modèle de référence pour les autres fédérations régionales :

«Yo llevo años acá con la Federación de Zacatecanos, y me impresionan, es algo muy bonito la manera que ayudan a su pueblo, pero también a veces me sorprendo cuando oigo : hay 300 Querétanos aqui, y 200 Jalicienses, me da gusto ver que hay mas grupos. Nos ayudan mucho, y a su comunidad también, con sus voluntarios, dan mucho para sus pueblos, como nosotros cuando colectamos fondos después del huracán y de la sequía en Zacatecas »?

12 En contrepartie, l'institutionnalisation s'est accrue avec la nomination d'un représentant officiel de l'État de Zacatecas aux États-Unis, l'ouverture d'un bureau de représentation et la promesse de faire élire deux députés migrants à l'assemblée de l'État. Ces mécanismes jouent également au plan local, puisque des maires de villages et villes de Zacatecas se rendent désormais en Californie pour faire campagne et, inversement, des migrants font campagne aux élections municipales mexicaines. Le cas le plus médiatisé est celui d'Andrés Bermudez, surnommé El Rey Tomate, qui a présenté sa candidature au poste d'édile de Jerez. Il a même remporté les élections avant d'être destitué faute de résidence permanente dans sa ville. Cette nouvelle dynamique politique a été décrite comme la naissance d'une élite migrante transnationale, facteur de démocratisation du système politique mexicain (Portes, 1996). En tout cas, l'intensification des rapports entre les élites politiques et les comités de village ou les fédérations régionales constitue le signe d'un rapprochement de l'état et des organisations, d'une centralisation des liens et d'une institutionnalisation des mécanismes de coopération.

13 L'intérêt renouvelé du Mexique envers sa diaspora tient à deux principaux facteurs. D'une part, le « Mexique de l'extérieur » représente un poids économique et politique important : 20 millions de Mexicains et Mexicains-Américains établis aux États-Unis, 9 millions d'électeurs potentiels, plus de 11 milliards de dollars de remises en 2003, des fédérations qui se multiplient, des lobbies potentiels aux États-Unis. Les mobilisations de certaines organisations aux États-Unis, pour réclamer le droit de vote au Mexique depuis l'étranger et l'aboutissement de la réforme constitutionnelle à cet effet, montrent la force acquise par la diaspora sur l'agenda des partis politiques mexicains (Le Texier, 2002 : 101-117 ; Castañeda, 2004 : 70-89). À cet égard, les campagnes répétées des gouverneurs des États les plus exportateurs de migrants et des présidents mexicains, en Californie et en Illinois, soulignent le poids politique qui leur est désormais attribué (Moctezuma, 2000). Ainsi, à la recherche de l'appui financier (Alarcón, 2002) et du soutien politique de la communauté migrante, de nombreux hommes politiques mexicains font désormais des États-Unis un passage obligatoire. Cuauhtémoc Cárdenas, du Parti de la Révolution Démocratique a été le premier à initier cette dynamique dès la fin des années 1980 . Il a été suivi par les candidats de tous les partis. La dernière campagne présidentielle de 2000 a ainsi vu les déplacements de Vicente Fox du Parti Action Nationale et de la femme de Francisco Labastida du Parti de la Révolution Institutionnelle (Fitzgerald, 2000). Les gouverneurs d'État à forte immigration ont aussi battu campagne dans le Sud-ouest américain, tels José Murat pour l'État de Oaxaca ou Ricardo Monreal pour l'État de Zacatecas.

14 D'autre part, la récupération par le haut de ces fédérations s'est aussi construite en réponse à un contexte de plus en plus hostile à l'immigration mexicaine aux États-Unis, où la militarisation de la frontière et l'adoption de législations restrictives visent à limiter «l'invasion» du pays par le sud. Le changement de politique étrangère 
mexicaine a donc pris la forme d'un «acercamiento» (rapprochement) avec ceux qui furent longtemps considérés comme des traîtres à la patrie. Selon Shain: "The manipulation of loyalty boundaries often extends beyond state borders to include diaspora members who may be discredited as outsiders, or alternatively be considered as insiders in accordance with the home government's changing view of them " (Shain, $2000: 663$ ). C'est ainsi que le gouvernement de Salinas de Gortari a mis en place le Programme pour les Communautés Mexicaines de l'Extérieur (PCME) en 1988. Créé sous l'égide du Parti de la Révolution Institutionnelle, le PCME marque une rupture du gouvernement mexicain à l'égard de la diaspora (Mohar, 2000 : 125-129). L'État d'origine encourage la solidarité internationale dans les deux sens : à partir de 1991, les migrants vont bénéficier d'une série de programmes d'aide dans le cadre du PRONASOLI, Programme National de Solidarité Internationale. Avec l'arrivée au pouvoir de Ernesto Zedillo, cette mutation se décline clairement en projet nationaliste. Le Programme de Développement National (1995-2000) stipule que « la Nation mexicaine s'étend au-delà du territoire délimité par ses frontières ». Par des moyens institutionnels et politiques, le gouvernement mexicain promeut la (ré)intégration de la communauté mexicaine de l'extérieur, soupape de sécurité économique et sociopolitique, dans la nation mexicaine. Inspiré du 2X1, le programme des Comités de Plan des États du Ministère du Développement Social et de l'office de représentation des Mexicains de l'extérieur, «Initiative Citoyenne $3 \mathrm{X} 1$ ", établit une coopération formelle entre les associations de village ou fédérations, l'État fédéral, l'État fédéré et la municipalité (Secretaría de Relaciones Exteriores, 2003).

15 La plus récente phase d'institutionnalisation des relations entre fédérations régionales et État mexicain est marquée par la naissance de l'Institut des Mexicains de l'Extérieur (IME). Cándido Morales, originaire de l'État de Oaxaca, connu pour son engagement associatif dans la communauté mexicaine aux États-Unis, est nommé à sa direction en octobre 2002. Il a procédé à la constitution du comité consultatif composé de Mexicains établis aux États-Unis : la première élection des représentants, très conflictuelle du fait des conditions de réalisation jugées souvent peu démocratiques (manque de temps, de transparence, procédures variant au plan local) a été menée en décembre 2002. Par la fondation de l'IME, le gouvernement de Vicente Fox cherche à renforcer la loyauté de la population immigrée envers le pays d'origine. Ainsi, le rôle des consulats sur le territoire américain devient encore plus crucial dans la consolidation et l'institutionnalisation des fédérations ${ }^{8}$. L'IME se donne aujourd'hui pour but de " fédérer les fédérations » et de dessiner les priorités de l'action conjointe en matière de protection des sans papiers, mais aussi des Mexicains résidents ou naturalisés, y compris de deuxième génération, et des binationaux. Pour Guarnizo : «transnational grassroots connections, traditionally built from below, are now being systematically formalized from above by nation-states of origin. This 'transnationalization from above' is much more extensive than it was a century ago, and it has far more reaching implications " (Guarnizo, 2001: 213-263). L'institutionnalisation des fédérations régionales incarne donc la promotion d'un nationalisme culturel au service des intérêts étatiques mexicains. Ainsi, le maintien de l'héritage culturel mexicain constitue un objectif prioritaire des représentants consulaires et des fédérations. Manuel de la Cruz Ramirez, représentant du gouvernement de l'État de Zacatecas aux États-Unis, confirme cette vocation à faire perdurer les traditions culturelles et linguistiques dans le pays d'accueil à travers les activités des fédérations :

"Queremos promover la cultura mexicana, porque la gente viene de México, se queda aquí y va perdiendo sus raíces, más bien su lazo con México, y con Zacatecas 
en particular, porque nosotros nos venimos todos de este lado. Nosotros promovemos la identidad cultural de los conacionales, con celebraciones, costumbres, deportes, artes, para todas las edades es importante, el 16 de septiembre, el Cinco de Mayo, estamos promoviendo todo $»^{9}$.

Les responsables consulaires tiennent un discours similaire, dont l'objectif nationaliste est tempéré par celui de favoriser l'ajustement des immigrés dans leur nouvelle société. Ainsi, la responsable de l'application du volet éducatif du Programme pour les Communautés Mexicaines de l'Étranger du consulat mexicain à San Diego affirme que la promotion de la culture d'origine constitue une réaction à la discrimination vécue aux États-Unis et contribue ainsi à former un capital social et culturel indispensable à l'adaptation des migrants dans la société d'accueil :

«El Programa para Comunidades Mexicanas en el Extranjero no es una reconquista, no hay idea de venganza, no, no creo... Es que ellos vienen aca y pierden sus raíces, es difícil vivir lejos de su país. Luego los niños nacen acá, se crian en los Estados Unidos, pero su físico siempre es Mexicano, y nunca son de acá. Tampoco cuando regresan a México son de allá, porque no crecieron allá. Asi hay que mantener el lazo, para que no se le corte su cultura al niño, que pueda aguantar el choque cultural, para su auto estima, es parte de su cultura que pueda usar para adaptarse $»^{10}$.

17 Thèmes de mobilisation pour les fédérations régionales mexicaines, enjeux de politique étrangère pour le gouvernement mexicain, les mesures anti-immigration ont donc alimenté la politique nationaliste mexicaine, hors de ses frontières géographiques. L'implication des États fédéraux et de l'État mexicain dans l'institutionnalisation ou la création des fédérations régionales mexicaines est une réponse à cette nouvelle situation. Les fédérations de Zacatecas, de Guerrero et du Michoacán ont signé des accords-cadres avec leurs gouvernements respectifs. Dans le cas de Guanajuato, l'État a mis en place une commission spéciale (Comisión Estatal de Apoyo Integral a los Migrantes y a sus Familias) ainsi qu'une direction générale pour les communautés de Guanajuato à l'Extérieur afin d'institutionnaliser la coopération. Le gouverneur de Jalisco a institué une journée annuelle pour les Jalisciences absents et soutenu des projets à investissements limités et des activités génératrices d'emplois dans le cadre du programme Por Mi Jalisco et d'un plan 3X1. De même, l'État du Michoacán a ouvert un bureau de liaison à Chicago (Coordinación General para la Atención al Migrante Michoacano) et noué des liens avec les leaders des comités de village. Enfin, dans le cas de la Fédération des Clubs de Michoacanos de Californie, comme dans celui de la Fédération de Zacatecanos, les gouverneurs des États ont entrepris de nommer un représentant officiel en Californie. Ces fédérations se présentent comme un cas typique d'une institutionnalisation qui participe du maintien de la conscience nationale des migrants et de leurs enfants. Ce n'est donc pas un hasard si les célébrations du Cinco de Mayo, de l'indépendance mexicaine ou du Día de los Muertos, sponsorisées par les consulats et les chambres de commerce, constituent des moments particuliers de recrutement et de collecte de fonds pour les fédérations. La formalisation des relations entre fédérations et États d'origine répond au revirement du gouvernement mexicain à l'égard de sa diaspora à la fin des années 1980. Dans une large mesure, le gouvernement mexicain, à travers ses consulats, le Programme pour les Communautés de l'Extérieur et plus récemment, l'Institut des Mexicains de l'Extérieur, est un élément primordial de l'institutionnalisation (et parfois en amont de la formation) des pratiques organisationnelles des migrants. 
18 Alors que la création d'associations de solidarité des migrants a permis un processus de transnationalisation par le bas, l'accompagnement institutionnel par le haut a renforcé les fédérations afin d'en contrôler le développement: les fédérations servent alors l'intérêt national mexicain tout autant que les intérêts transfrontaliers de ses membres. Toutefois, si la majorité des fédérations sont entrées dans une phase de consolidation, d'autres continuent de revendiquer une forme d'activisme transnational par le bas. Il existe une différence fondamentale entre les fédérations institutionnalisées, souvent mestizas, et les fédérations autonomes, indigènes. Ces dernières conçoivent leur action en opposition au projet d'institutionnalisation étatique et développent une identité collective indigène distincte du projet nationaliste mexicain.

\section{Les fédérations indigénistes, une résistance au projet nationaliste}

19 Pour les fédérations indigènes, la construction d'une communauté migrante transnationale peut être appréhendée comme une réaction à une exclusion ressentie et vécue tant dans l'État d'origine que d'accueil. Dans ce cadre, les migrants indigènes de Oaxaca, tels ceux de la Federación Oaxaqueña (FO), mais aussi de la Coalición de Comunidades Indígenas de Oaxaca (COCIO) ou du Frente Indígena Oaxaqueño Binacional (FIOB) s'engagent dans des activités transfrontalières en réaction à une double discrimination. Comme le dit José González, président de la section de Oceanside du FIOB, la migration implique un engagement solidaire dédoublé, mais permet aussi de revendiquer des droits des deux côtés de la frontière :

«Soy doble nacional. Opino que necesitamos hacer un cambio para que se mejore la situación en Oaxaca y México, y puedo participar para cambiar las cosas allá. También soy ciudadano americano acá, acá trabajo y pago taxas, y quiero que se respeten los derechos de mi comunidad ${ }^{11}$.

Ainsi, à l'identité collective nationale impulsée par le gouvernement mexicain, répond une action collective qui revendique la défense des intérêts indigènes des deux côtés de la frontière : «It is when exit and voice options are blocked both in country of origin and country of destination that the transnational field becomes an attractive field of activity that enables migrants to combine what is advantageous about their origins and their destinations » (Roberts, Frank et Lozano-Ascensio, 1999 : 253).

21 La ressource identitaire fournit alors le cadre d'une résistance à l'institutionnalisation. Les fédérations indigènes visent à obtenir des bénéfices matériels et une reconnaissance de leur spécificité dans les deux pays. Ainsi, alors que les fédérations mestizas de Zacatecas, Guanajuato ou Jalisco renforceraient l'attachement nationaliste à la patrie mexicaine, les comités et fédérations de Oaxaca développeraient une identité collective indigéniste. Certes, les organisations régionales indigènes ne diffèrent que peu des autres fédérations dans leur structure. Elles sont d'abord des organisations à but non lucratif, mutualisant les ressources en vue d'exercer des activités philanthropiques. Mais elles se distinguent par l'incorporation de pratiques communautaires indigènes propres, qui peuvent impliquer le retour, même contre leur gré, des migrants dans leur village d'origine. Par exemple, le système du tequio (travail d'utilité collective), des cargos (position de leadership communautaire par rotation de pouvoir) et le respect du droit coutumier (prise de décisions consensuelle, après réunion de l'assemblée communautaire) induisent des rapports très denses avec la 
communauté d'origine. Rufino Domínguez Santos, coordinateur général du FIOB, insiste sur l'importance des liens sociaux communautaires qui fondent l'autonomie de l'organisation et sur sa spécificité indigène ${ }^{12}$. Le développement de communautés migrantes indigènes transnationales, mixtèque ou zapotèque, tiendrait alors à trois phénomènes : le caractère relativement récent des migrations indigènes en provenance de l'État de Oaxaca; la constitution d'organisations communautaires solides; les spécificités culturelles et sociales de ces migrants. Comme l'indiquent Rivera-Salgado et Escala (2002:8): «The result of this is that on many occasions the migrants maintain strong translocal ties, even to the point in which participation in and the structure of compatriot associations is strongly regulated by local authorities in the community of origin, who send official designations to people who live thousands of miles away ». À l'inverse des fédérations mestizas, les fédérations indigènes refusent tant l'institutionnalisation étatique que la détermination des activités par la seule communauté migrante de Californie. Le cas du FIOB illustre cette double dynamique de résistance au projet nationaliste et d'intégration des spécificités des communautés indigènes.

Contrairement aux autres fédérations régionales, le FIOB tire son origine d'une logique d'opposition à l'État mexicain, basée sur une revendication politique : l'exigence de démocratie et d'égalité des droits des populations indigènes au Mexique. Le nom de l'organisation est d'ailleurs symbolique, c'est un Front, et pas une fédération, même si elle en adopte les statuts et le fonctionnement. Créé en 1985 d'abord à Oaxaca, le Front Civique Mixtèque s'est exporté en Californie dès 1989, incluant la population Zapotèque de Oaxaca dans le Front Civique Mixtèque Zapotèque, puis devenant en 1990 le Front Binational des Indigènes de Oaxaca. Le FIOB s'est étendu avec, entre autres, des branches à Los Angeles, Fresno, Oceanside. Il promeut la défense des indigènes de Oaxaca, en particulier en luttant pour les droits des travailleurs agricoles, la protection des illégaux et la promotion de mesures anti-discriminatoires aux États-Unis et au Mexique (Moctezuma, 2000). Le FIOB se définit comme négociateur, intermédiaire et représentant d'un niveau alternatif de pouvoir dans un nouvel espace transnationalisé. En effet, la transnationalisation des activités de solidarité fournit aux migrants indigènes des compensations matérielles et symboliques, mais reflète surtout la résistance aux autorités locales et étatiques. Du côté des rétributions matérielles, la dynamique économique de coopération constitue un capital financier et social qui favorise l'émergence d'une classe de petits entrepreneurs indigènes transnationaux. De même, du côté des rétributions symboliques, les migrants accroissent leur position du fait de l'éventuelle ascension sociale vécue dans l'immigration. À la discrimination ressentie dans le pays d'origine de la part des populations mestizas se substitue parfois un reclassement social des indigènes dans la migration. La transnationalisation des activités des migrants indigènes permet d'inverser la hiérarchie traditionnelle dominée par l'élite métisse ou blanche et de lutter contre la discrimination ressentie. José, immigré depuis 18 ans, naturalisé américain, travailleur du secteur des services exprime son engagement au sein du FIOB en réaction à cette position d'infériorité statutaire :

"A los que estamos en los USA, nos llaman "Los del Norte", en México nos llaman Indios o indígenas, en los Estados Unidos, los demás trabajadores nos llaman indígenas. Es el mismo problema de la discriminación por todas partes, son iguales las injusticias, de su propia gente, no se respetan los derechos laborales y humanos, igual aca y en Oaxaca, a los indígenas se les pone a trabajar, no tienen poderes, les tratan como los esclavos. Es que en Oaxaca, es dificil vivir, por el trabajo, estás 
humillado siempre por la gente mestiza (...). Eres indígena, y te consideran que eres de cuarta clase $»^{13}$.

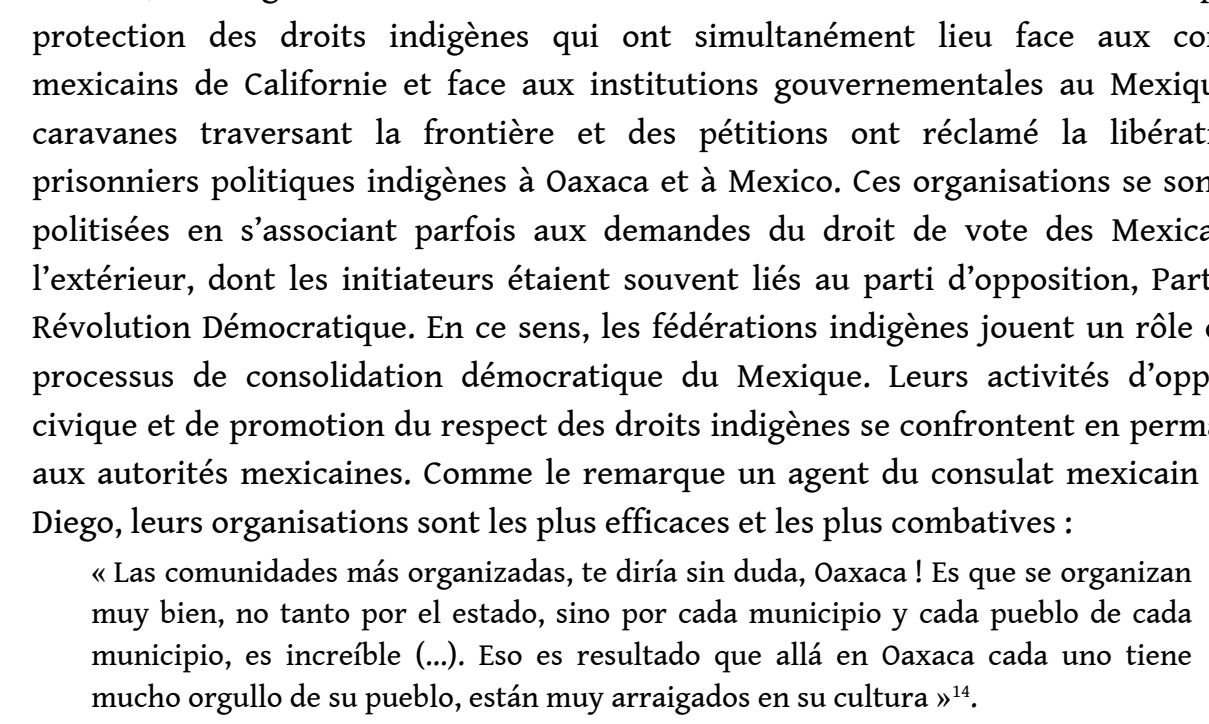

Le refus de la cooptation par le haut se lit ensuite dans le fractionnement fort des fédérations indigènes de Oaxaca en Californie. Ce n'est que très récemment que la Federación Oaxaqueña a vu le jour, et sa légitimité n'est pas entièrement reconnue par d'autres fronts, fédérations ou collectifs d'associations indigènes. La résistance à une organisation univoque qui chapeauterait l'ensemble des associations illustre certes les divisions de la communauté indigène de Oaxaca, sur des bases ethniques, politiques ou stratégiques, mais aussi une structuration qui se veut autonome des logiques des partis politiques mexicains qui ont tissé des liens de plus en plus importants avec leurs représentants et des autorités mexicaines. Dans tous les cas, les fédérations mexicaines, institutionnalisées ou de résistance, se caractérisent par leur capacité de mobilisation et de négociation dans les deux pays, tant au plan local que national. Acteurs politiques transnationaux, les fédérations tentent de déterminer les politiques entreprises tant par l'État d'origine que d'accueil, même si les résultats de la mobilisation sur les enjeux américains restent encore limités (Zabin et Escala, 2002 : 7-42). Elles sont devenues de nouveaux acteurs de la politique étrangère mexicaine et des relations bilatérales entre le Mexique et les Etats-Unis, acteurs avec lesquels il faut désormais compter. 


\section{BIBLIOGRAPHIE}

ALARCóN Rafael (1988) El proceso de norteñización : impacto de la migración internacional en Chavinda, Michoacán, in Thomas Calvo et Gustavo López Éds., Movimientos de población en el occidente de México, México D.F., El Colegio de Michoacan, pp. 43-59.

BASCH Linda, SCHILLER Nina et BLANC-SZANTON Cristina (1994) Nations Unbound: Transnational Projects, Post-Colonial Predicaments and Deterritorialized Nation States, Langhorne, Gordon and Breah, $344 \mathrm{p}$.

BASCH Linda, SCHILLER Nina et BLANC-SZANTON Cristina Éds. (1995) Towards a Transnational Perspective on Migration : Race, Class, Ethnicity, and Nationalism Reconsidered, Annals of the New York Academy of Sciences, 645 (2), pp. 25-52.

CASTAÑEDA Alejandra (2004) Roads to Citizenship : Mexican Migrants in the United States, Latino Studies, 2, pp. 70-89.

DELAUNAY Daniel et LESTAGE Françoise (1999) Des histoires de vie, une histoire de famille. Ménages et fratries des Mexicains aux États-Unis, Revue Européenne des Migrations Internationales, (15) 3, pp. 11-43.

DOMINGUEZ SANTOS Rufino (2002) La experiencia del FIOB, crisis interna y futuros retos, Indigenous Mexican Migrants in the US : Building Bridges Between Researchers and Community Leaders, Santa Cruz, UCSC Conference, http://www.lals.ucsc.edu/conference

FARET Laurent (2003) Les territoires de la mobilité. Migration et communautés transnationales entre le Mexique et les États-Unis, Paris, Editions du CNRS, 351 p.

FITZGERALD David (2000) Negotiating Extra-Territorial Citizenship. Mexican Migration and the Transnational Politics of Community, La Jolla, CCIS Monograph n $2-$ UCSD, 138 p.

GARDUÑO Everardo (2003) Antropología de la frontera, la migración y los procesos transnacionales, Frontera Norte, 15 (30), pp. 65-89.

GUARNIZO Luis (2001) On the Political Participation of Transnational Migrants : Old Practices and New Trends, in Gary Gerstle et John Mollenkopf Éds., E Pluribus Unum ? Contemporary and Historical perspectives on Immigrant Political Incorporation, New York, Russell Sage Foundation, pp. 213-263.

GUARNIZO Luis et SMITH Michael Éds. (1998) Transnationalism from below, New Brunswick, Transactions Publishers, 316 p.

GUTIÉRREZ David Éd. (1996) Between Two Worlds. Mexican Immigrants in the United States, Wilmington, Jaguar Books, 271 p.

KEARNEY Michael (1995) The effects of Transnational Culture, Economy, and Migration on Mixtec Identity in Oaxacalifornia, in Michael Smith et Joe Feagin Éds., The Bubble Cauldron : Race, Ethnicity, and the Urban Crisis, Minneapolis, University of Minnesota Press, pp. 23-45.

LE TEXIER Emmanuelle (2002) Les Mexicains des États-Unis et la revendication du droit de vote au Mexique : des élections de 1988 aux présidentielles 2000, Cahier des Amériques Latines, 37 (2), pp. 101-117.

LE TEXIER Emmanuelle (2004) Immigration et obsession sécuritaire aux États-Unis, Questions Internationales, 5, pp. 88-96. 
MASSEY Douglas et GOLDRING Luin (1994) Continuities in Transnational Migration : An Analysis of Nineteen Mexican Communities, American Journal of Sociology, 99 (6), pp. 1492-1533.

MASSEY Douglas et ALARCÓN Rafael (1987) Return to Aztlán : The Social Process of International Migration from Western Mexico, Berkeley, University of California Press, 335 p.

MASSEY Douglas, DURAND Jorge et MALONE, Nolan (2002) Beyond smoke and mirrors: Mexican immigration in an era of economic integration, New York, Russell Sage, 199 p.

MOCTEZUMA Miguel (2000) Clubes de Migrantes, Expresión Organizada del Migrante Colectivo, in Cuauhtémoc Cárdenas Batel et Gonzalo Badillo Moreno Éds., Los Derechos de los Migrantes en los Estados Unidos, México D.F., Instituto de Investigaciones Legislativas Cámara de Diputados, 454 p.

MOCTEZUMA Miguel (2000) El circuito migrante Sain Alto, Zac. - Oakland, Ca., Comercio Exterior, 50 (5), pp. 396-405.

MOHAR Gustavo (2000) Relations with the Mexican Diaspora, in Rodolfo De La Garza et Harry Pachón Éds., Latinos and U.S. Foreign Policy. Representing the "Homeland" ?, Boulder, Rowman \& Littlefield, pp. 125-129.

ODGERS Olga (2003) Flux migratoires du Mexique vers les Etats-Unis : changement et continuité, Problèmes d'Amérique latine, 50, pp. 59-78.

OROZCO Manuel et LAPOINTE Michelle (2003) Mexican Hometown Associations and Their development Opportunities, Report commissioned by the U.S. Agency for International Development, InterAmerican Dialogue, Research Series on Remittances n³, http://www.thedialogue.org

PORTES Alejandro (1996) Transnational communities : their emergence and significance in the contemporary world system, in Roberto Korzeniewicz et William Smith Éds., Latin American and the World Economy, New York, Greenwood Press, pp. 151-168.

RIVERA-SALGADO Gaspar (2000) Migration and political activism : Mexican transnational indigenous communities in a comparative perspective. Thesis University of California Santa Cruz, Ann Arbor, UMI Dissertation Services, 390 p.

RIVERA-SALGADO Gaspar et ESCALA RABADÁN Luis (2002) Collective Identity and Organizational Strategies Among Indigenous and Mestizo Mexican Migrants, Indigenous Mexican Migrants in the US : Building Bridges Between Researchers and Community Leaders, Santa Cruz, UCSC Conference, http://www.lals.ucsc.edu/conference

ROBERTS Bryan, FRANK Reanne et LOZANO-ASCENSIO Fernando (1999) Transnational Migrant Communities and Mexican Migration to the US, Ethnic and Racial Studies, 22 (2), pp. 238-266.

SECRETARÍA DE RELACIONES EXTERIORES (2003) Programa para las comunidades mexicanas en el Extranjero - Program for Mexican Communities Abroad, México D.F., SRE, 35 p.

SHAIN Yossi (2000) The Mexican-American Diaspora's Impact on Mexico, Political Science Quarterly, 14 (4), pp. 661-691.

U.S. CENSUS BUREAU (2001) Overview of Race and Hispanic Origin, U.S Department of Commerce, March 2001, http://www.census.gov

ZABIN Carol et ESCALA RABADÁN Luis (2002) From Civic Association to Political Participation : Mexican Hometown Associations and Mexican Immigrant Political Empowerment in Los Angeles, Frontera Norte, 14 (27), pp. 7-42. 


\section{NOTES}

1. En 1994, l'opération Gatekeeper a été mise en place pour « sécuriser et protéger les frontières extérieures des États-Unis par la prévention des entrées illégales et par la détection, l'interdiction et l'appréhension des personnes entrant sans papiers, des passeurs, des contrebandiers, et toute personne violant les lois ». Elle a été suivie de l'opération Hold the Line, Rio Grande et Safeguard au Texas et en Arizona.

2. «Prior empirical work suggests that transnational migration unfolds in a relatively consistent way of times. It displays a distinct tendency to become more prevalent and to broaden its base of demographic, social and economic representation within the community. These trends follow theoretically from the fact that migration affects individual motivations and social structures in ways that encourage additional migration. As a result, transnational migration tends to become a self-reinforcing process that acquires an internal momentum on its own. Over time it becomes increasingly independent of the conditions that originally caused it » (Massey et Goldring, 1994 : 1496).

3. Cet article se fonde sur un matériau empirique collecté de septembre 2002 à février 2004 en Californie: d'une part des entretiens menés avec des membres de comités de village et de fédérations régionales à San Diego, Los Angeles et San José, et d'autre part, l'observation de réunions de la Fédération de Zacatenos, du Frente Indígena Oaxaqueño Binacional, de la Fédération des Clubs de Michoacan et de celle du Guerrero. L'échantillon est limité à la Californie et à quatre Fédérations. (Traductions de l'espagnol au français par l'auteure).

4. Le registre de ces associations auprès des consulats mexicains est aléatoire. Une association de village peut regrouper en moyenne de 3 à 30 membres. En Californie, elles sont concentrées à Los Angeles. Selon Gustavo Cano, de l'Institut des Mexicains de l'Etranger (IME), le développement des fédérations mexicaines dépend fortement de la densité des réseaux migratoires. Ainsi, il y aurait à Chicago 160 associations de village et 7 fédérations (Durango, Guanajuato, Guerrero, Jalisco, Michoacán, San Luis Potosi et Zacatecas), mais seulement 20 clubs de village et une fédération (Zacatecas) à Houston. Entretien, 12 avril 2003.

5. «C'est pour cela que je travaille pour le développement de Santa Rosa, c'est un village qui ne compte plus que 500 habitants, parce que 1400 sont venus de ce coté-ci. Depuis que nous avons créé le comité, on envoie de l'argent et des programmes, on a fait installer l'eau potable, l'éclairage public, on a réalisé $95 \%$ des routes, c'est nous qui les avons toutes asphaltées. C'est parce que le gouvernement ne remplit pas ses promesses et ses devoirs que la coopération existe entre le village de Santa Rosa et nous ».

6. Voir, entre autres, les exemples de Chavinda, Michoacán et San José, Californie (Alarcón, 1988) ; Aguililla, Michoacán et Redwood City, Californie (Rouse, 1991) ; Ticuani, Puebla et New York, New Jersey (Smith, 1998) ; México D.F. et Austin, Texas (Roberts, Frank et Lozano-Ascensio, 1999: 238-266) ; Sahuayo, Michoacán et Santa Ana, Californie (Fitzgerald, 2000); Sain Alto, Zacatecas et Oakland, Californie (Moctezuma, 2000 : 396-405). Pour une vue d'ensemble : (RiveraSalagado, 2000).

7. «Cela fait desannées que je suis à la Fédération de Zacatecanos, (ses membres) m'impressionnent, c'est vraiment bien l'action qu'ils mènent pour aider leurs compatriotes. Mais cela me surprend aussi d'entendre qu'il y a 300 Querétanos ici et 200 Jalicienses là. Cela me fait plaisir de voir qu'il existe d'autres groupes. Ils nous aident beaucoup comme ils aident leur communauté, ce sont des bénévoles qui font beaucoup pour leurs compatriotes, comme nous lors de la collecte de fonds après la tempête et la sécheresse à Zacatecas ".

8. Pour l'historien Gutiérrez (1996: XIII), la vocation des consultas n'a rien de nouveau : «For its part, Mexico's government has been deeply ambivalent about the mass migration of its citizens to the United States (...). Thus, since the 1910 revolution, Mexico's emigration policy traditionally has been predicated on attempting to protect the rights of its citizens abroad (largely through diplomatic channels and through the efforts of the consular corps in the United States); maintaining a sense of patriotism 
among expatriates by sponsoring observances of national holidays in ethnic Mexican communities in the United States; and encouraging, and a times actively fostering, the eventual repatriation of Mexican nationals abroad ».

9. «Nous voulons promouvoir la culture mexicaine, parce que les gens viennent du Mexique, ils s'établissent ici, et ils perdent progressivement leurs racines, leur lien avec le Mexique, et en particulier avec Zacatecas, parce que nous sommes tous venus de ce côté-ci. Alors nous promouvons l'identité culturelle des compatriotes, avec des célébrations, les coutumes, les sports, les arts, quel que soit l'âge c'est important, le 16 septembre, le 5 mai, on célèbre tout ».

10. «Le Programme pour les Communautés Mexicaines de l'Extérieur n'est pas une reconquête, il n'y a pas d'idée de vengeance, non, je ne crois pas... Le fait est qu'ils (les migrants) arrivent ici et perdent leurs racines, c'est dur de vivre loin de son pays. Ensuite, leurs enfants naissent ici, sont élevés aux États-Unis, mais physiquement, ils restent des Mexicains et ils ne sont jamais vraiment d'ici. Ils ne peuvent pas non plus rentrer au Mexique, parce qu'ils n'y ont pas grandi. Donc il faut maintenir le lien, pour que l'enfant connaisse sa culture, qu'il puisse supporter le choc culturel, pour l'estime de soi. Préserver une partie de sa culture qu'il puisse utiliser pour s'adapter ».

11. «Je suis binational. Je pense que nous devons provoquer un changement pour que s'améliore la situation à Oaxaca et au Mexique et je peux participer à ce changement là-bas. Mais je suis aussi citoyen américain ici, c'est ici que je travaille et que je paie mes impôts, et je veux qu'ici on respecte les droits de ma communauté ».

12. "La solidarité, le lien avec les Oaxaqueños, ne se limite pas à ceux qui vivent aux États-Unis, mais a une dimension binationale. La preuve, c'est qu'à Oaxaca, on a pu nommer des Oaxaqueños qui résident au États-Unis pour participer aux réunions avec leurs compatriotes sur le tequio et l'ensemble des enjeux de développement de la communauté » (Domínguez Santos, 2002 : 2).

13. «Nous qui vivons aux États-Unis, on nous appelle Ceux du Nord. Au Mexique, on nous appelle Indiens ou Indigènes ; aux États-Unis, les autres travailleurs nous appellent indigènes. Le problème de la discrimination est le même partout, ce sont les mêmes injustices, y compris de la part de ses compatriotes. On ne respecte pas le droit du travail, ni les droits de l'Homme, ici et à Oaxaca, c'est la même chose. On fait travailler les indigènes, sans respecter leurs droits, comme des esclaves. C'est difficile de vivre à Oaxaca, au travail, tu es toujours humilié par les métisses (...). Si tu es indigène, tu es de quatrième classe ».

14. "Les communautés les mieux organisées, sans aucun doute, ce sont celles de Oaxaca! Ils s'organisent vraiment bien, pas seulement au plan régional, mais aussi pour chaque municipio, et chaque village de chaque municipio. C'est incroyable. (...) Cela vint du fait que là-bas à Oaxaca, chacun est très fier de son village, ils sont très enracinés dans leur culture »

INDEX

Mots-clés : associations, identité collective, Mexicains, participation politique 


\section{AUTEUR}

\section{EMMANUELLE LE TEXIER}

Doctorante à l'IEP de Paris, rattachée au CERI, Marie Curie Fellow au Centre d'Études de l'Ethnicité et des Migrations. Université de Liège - Bat. 31, Boîte 38, 7, Bvd du rectorat - B-4000 Liège (Sart Tilman), Belgique.eletexier@ucsd.edu 